[Supplementary Materials for:]

\title{
Chromium(III)(Salen)/DMAP: An Efficient Catalyst System for the Selective Synthesis of 5- Substituted Oxazolidinones from Carbon Dioxide and Aziridines
}

\author{
Aaron W. Miller and SonBinh T. Nguyen \\ Department of Chemistry and Institute for Environmental Catalysis, Northwestern University, 2145 Sheridan Rd., \\ Evanston, IL 60208-3113(USA).Fax:847-491-7713,Email: stn@chem.northwestern.edu.
}

General Information. ${ }^{1} \mathrm{H}$ and ${ }^{13} \mathrm{C}$ NMR spectra were recorded on either a Varian Inova $500\left(499.570 \mathrm{MHz}\right.$ for ${ }^{1} \mathrm{H}$, $125.631 \mathrm{MHz}$ for $\left.{ }^{13} \mathrm{C}\right)$ or a Mercury $400\left(400.168 \mathrm{MHz}\right.$ for ${ }^{1} \mathrm{H}, 100.631 \mathrm{MHz}$ for $\left.{ }^{13} \mathrm{C}\right)$ spectrometer. NMR data are reported as follows: chemical shift (multiplicity $(\mathrm{b}=$ broad, $\mathrm{s}=$ singlet, $\mathrm{d}=$ doublet, $\mathrm{t}=$ triplet, $\mathrm{q}=$ quartet, and $\mathrm{m}=$ multiplet), and integration). ${ }^{1} \mathrm{H}$ and ${ }^{13} \mathrm{C}$ chemical shifts are reported in ppm downfield from tetramethylsilane (TMS, $\delta$ scale) with the solvent resonances as internal standards. IR spectra were collected on a Nicolet 5PC instrument and analyzed using PC-IR software. High-Resolution Electron-impact Mass Spectra (HREIMS) were obtained from the Analytical Services Laboratory, Northwestern University (Evanston, IL). Elemental analyses were provided by Atlantic Microlab, Inc. (Norcross, GA). GC analyses of oxazolidinone products were carried out on a Hewlett Packard 5890A gas chromatograph equipped with FID detector and a 30-m HP-5 capillary column with 0.32-mm inner diameter and 0.25-mm film thickness was used. Temp program: intitial time $=0 \mathrm{~min}$., initial temperature $=60^{\circ} \mathrm{C}$, rate $=20^{\circ} \mathrm{C} / \mathrm{min}$; final time $=$ 2.5 min., final temperature $=250^{\circ} \mathrm{C}$.

Materials. Catalyst was synthesized according to published procedures. ${ }^{1,2}$ Racemic diaminocyclohexane was obtained from Aldrich. Solvents used were purchased from either Fischer Chemical or Aldrich Chemical. Deuterated solvents were purchased from Cambridge Isotope Laboratories and used without further purification. All other reagents were purchased from the Aldrich Chemical Company and used without further purification, unless otherwise noted. Aziridines and $\beta$ amino alcohol precursors were synthesized using published procedures. ${ }^{3}$ Data are available for 3,5diphenyloxazolidinone. ${ }^{4}$ Analytical data for the following compounds has not been reported and was confirmed by us.

General experimental procedure. On the bench top, a 45 -mL Parr high pressure reactor equipped with a magnetic stir bar was charged with catalyst $1\left(12.6 \mathrm{mg}, 2 \times 10^{-5} \mathrm{~mol}\right)$, DMAP $\left(4.9 \mathrm{mg}, 4 \times 10^{-5} \mathrm{~mol}\right)$, and a solution of the aziridine $(2 \mathrm{mmol})$ in $\mathrm{CH}_{2} \mathrm{Cl}_{2}$ (4 mL, $0.5 \mathrm{M}$ solution). Finally, undecane (100 $\mu \mathrm{l}, 0.474 \mathrm{mmol}$, internal standard) was placed in the reactor. The reactor was sealed and placed under constant $\mathrm{CO}_{2}$ pressure for 5 minutes to allow equilibration, the $\mathrm{CO}_{2}$ valve was closed, and the reactor was placed in a magnetically stirred $100{ }^{\circ} \mathrm{C}$ oil bath. After $2 \mathrm{~h}$ the reactor was removed from the oil bath, quickly cooled in running cold tap water, and vented to a hood. A small aliquot was then removed from the solution for $\mathrm{GC}$ analysis. (The catalyst was removed by eluting the aliquot in $\mathrm{CH}_{2} \mathrm{Cl}_{2}(20 \mathrm{~mL})$ through a solvent-wet silica plug which was doped with triethylamine $(100 \mu \mathrm{L})$ before introduction of the aliquot. Yield was determined via GC using peak areas and undecane internal standard.). Further purification by column chromatography over neutral alumina, (150 mesh, 58Å, hexanes:ethyl acetate 60:40) gave analytically pure oxazolidinone product (mixture of 4- and 5-substituted isomers).

$N$-propyl-5-phenyloxazolidinone. ${ }^{1} \mathrm{H} \mathrm{NMR}\left(\mathrm{CDCl}_{3}, 500 \mathrm{MHz}\right): \delta 0.97$ (t, $3 \mathrm{H}$, propyl $\left.\mathrm{CH}_{3},{ }^{3} \mathrm{~J}=7.0 \mathrm{~Hz}\right), 1.63(\mathrm{q}, 2 \mathrm{H}$, propyl $\left.\mathrm{CH}_{2},{ }^{3} \mathrm{~J}=7.0 \mathrm{~Hz}\right), 3.29\left(\mathrm{~m}, 2 \mathrm{H}\right.$, propyl $\left.\mathrm{N}-\mathrm{CH}_{2}\right), 3.47\left(\mathrm{t}, 1 \mathrm{H}, \mathrm{N}-\mathrm{CH}_{2},{ }^{3} \mathrm{~J}=8.0 \mathrm{~Hz}\right), 3.94\left(\mathrm{t}, 1 \mathrm{H}, \mathrm{N}-\mathrm{CH}_{2},{ }^{3} \mathrm{~J}=8.5 \mathrm{~Hz}\right)$, $5.53\left(\mathrm{t}, 1 \mathrm{H}, \mathrm{O}-\mathrm{CH},{ }^{3} \mathrm{~J}=8.0 \mathrm{~Hz}\right), 7.40(\mathrm{~m}, 5 \mathrm{H}$, arom- $H) .{ }^{13} \mathrm{C}\left\{{ }^{1} \mathrm{H}\right\} \operatorname{NMR}\left(\mathrm{CDCl}_{3}, 125 \mathrm{MHz}\right): \delta 11.3$ (propyl $\left.\mathrm{CH}_{3}\right), 20.8$ (propyl $\mathrm{CH}_{2}$ ), 45.9 (propyl $\left.\mathrm{CH}_{2}\right), 52.2\left(\mathrm{~N}_{-} \mathrm{CH}_{2}\right), 74.5(\mathrm{O}-\mathrm{CH}), 125.7(\mathrm{Cm}), 128.9(\mathrm{Co}), 129.0(\mathrm{Cp}), 139.2(\mathrm{Ci}), 158.2$ $(C=\mathrm{O})$. FTIR $\left(\mathrm{CH}_{2} \mathrm{Cl}_{2}\right)$ : $\mathrm{v}_{\mathrm{CO}} 1752.55 \mathrm{~cm}^{-1}$. Anal.: Calcd for $\mathrm{C}_{12} \mathrm{H}_{15} \mathrm{NO}_{2}$ : C, 70.22; $\mathrm{H}, 7.37 ; \mathrm{N}, 6.82$; Found: $\mathrm{C}, 70.15, \mathrm{H}$, 7.38, N, 6.87. HREIMS: Calcd for $\mathrm{C}_{12} \mathrm{H}_{15} \mathrm{NO}_{2}$ : 205.1097, Found: 205.1097. 
Figure S1. $\quad$ A) The ${ }^{1} \mathrm{H}$ spectrum of $N$-propyl-5-phenyloxazolidinone. There is a small amount of the 4-substituted isomer in this sample.

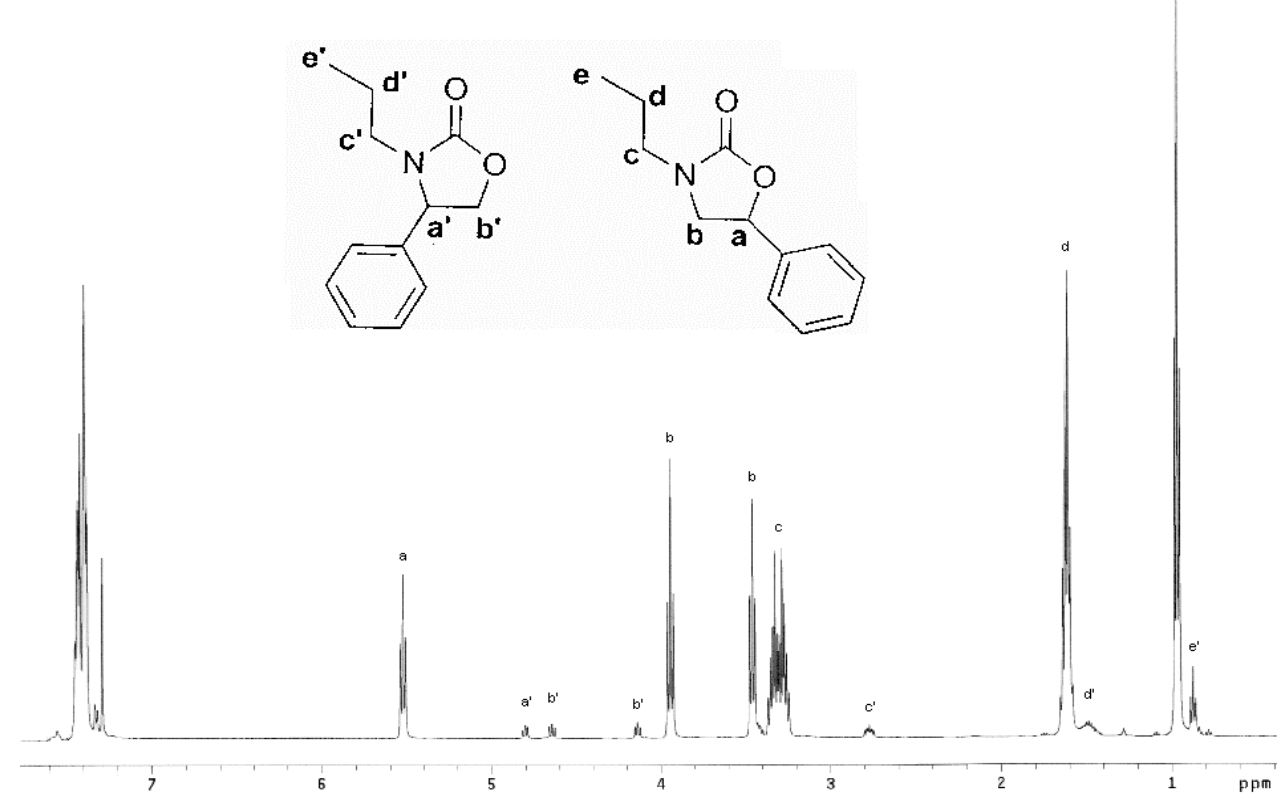

B) The NOESY spectrum of $N$-propyl-5-phenyloxazolidinone.

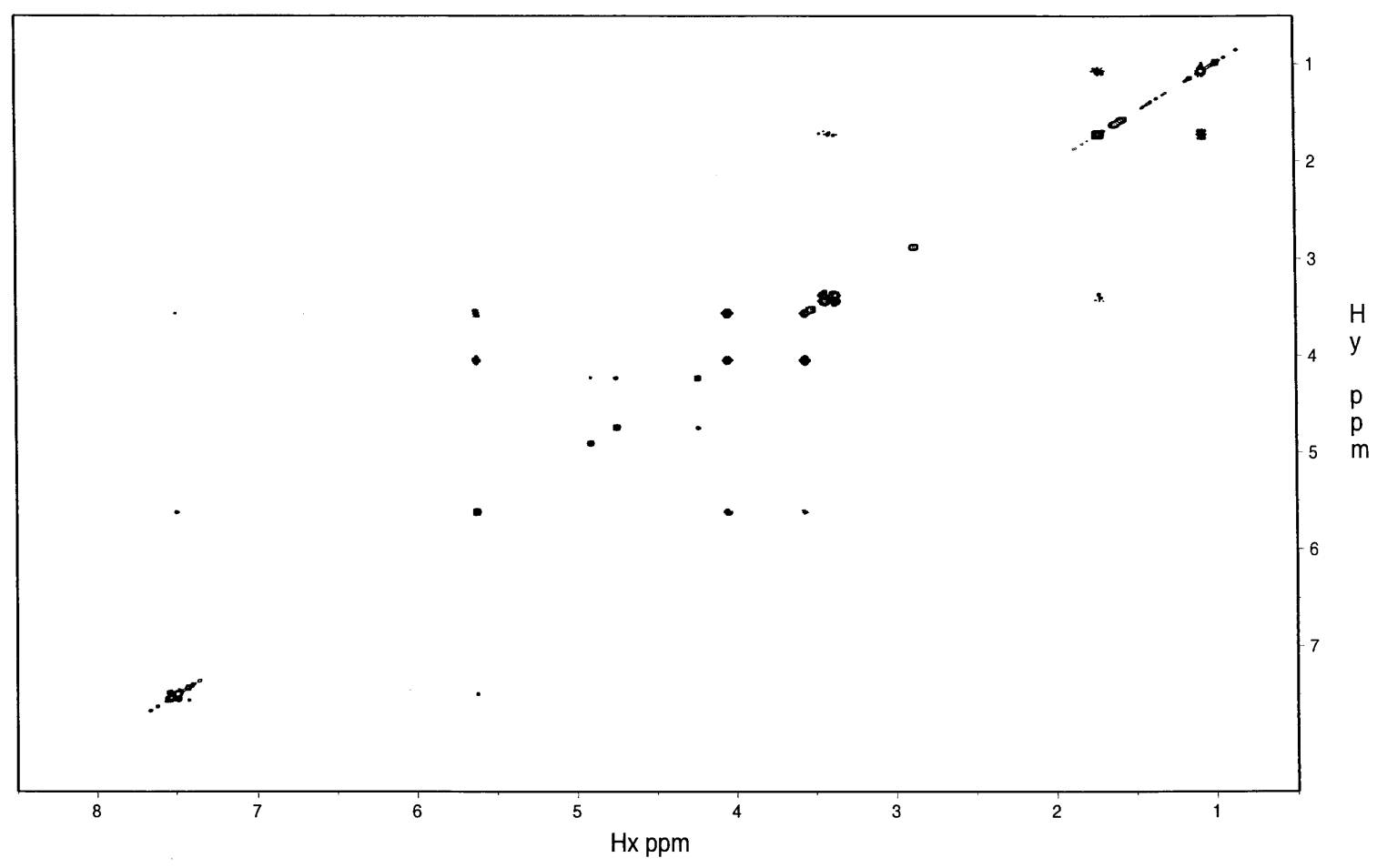


C) The FT-IR Spectrum of $N$-propyl-5-phenyloxazolidinone.

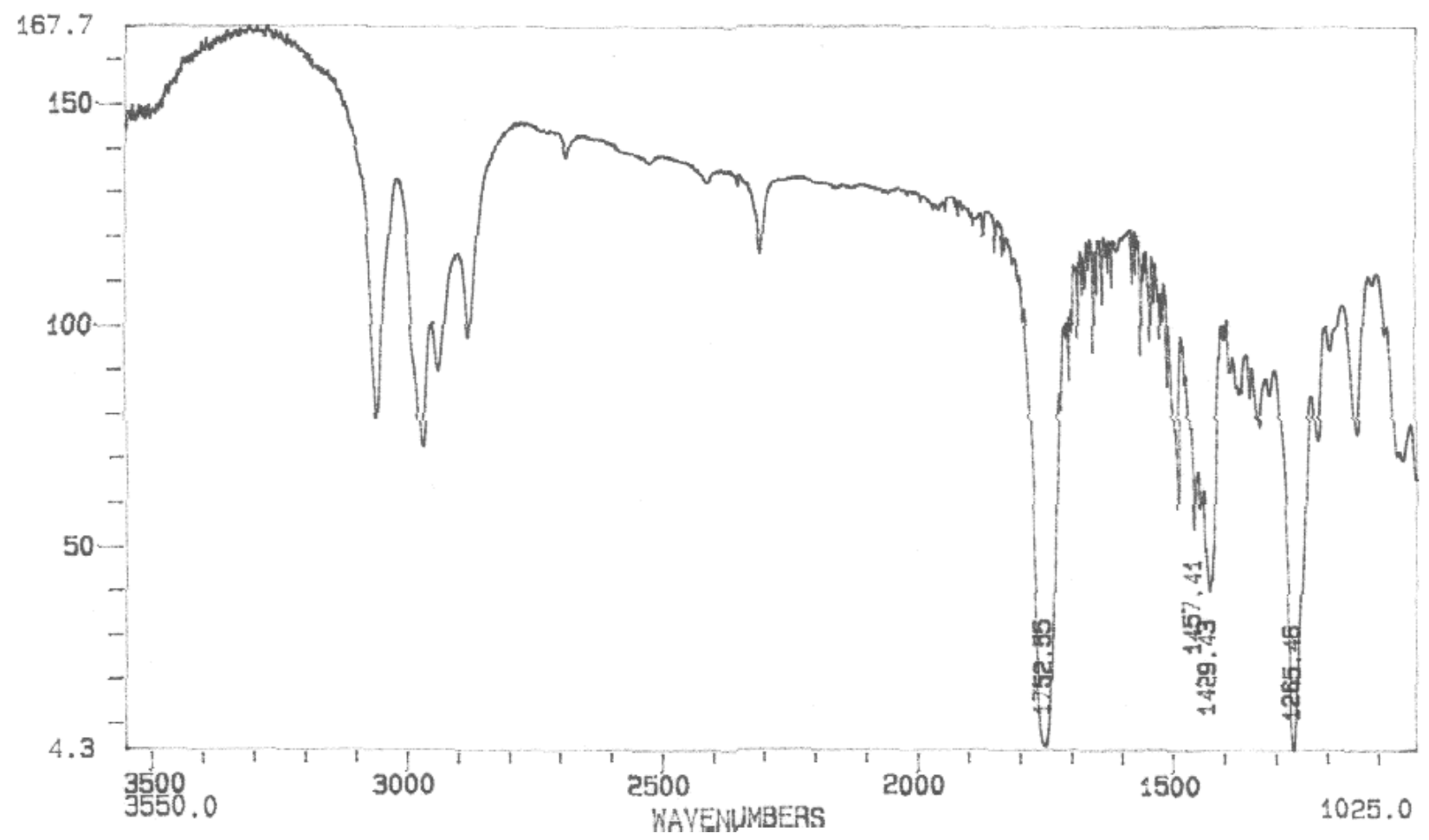

$N$-hexyl-5-phenyloxazolidinone. A yellow solid (mp 31.0-31.9 $\left.{ }^{\circ} \mathrm{C}\right) .{ }^{1} \mathrm{H} \mathrm{NMR}\left(\mathrm{CDCl}_{3}, 500 \mathrm{MHz}\right): \delta 0.86\left(\mathrm{t}, 3 \mathrm{H},{ }^{n}\right.$ hexyl $\left.\mathrm{CH}_{3},{ }^{3} \mathrm{~J}=6.0 \mathrm{~Hz}\right), 1.28\left(\mathrm{~m}, 6 \mathrm{H},{ }^{n}\right.$ hexyl $\left.\mathrm{CH}_{2}\right), 1.53\left(\mathrm{~m}, 2 \mathrm{H},{ }^{n}\right.$ hexyl $\left.\mathrm{CH}_{2}\right), 3.28\left(\mathrm{~m}, 2 \mathrm{H}, \mathrm{N}-\mathrm{CH}_{2}\right), 3.41\left(\mathrm{dd}, 1 \mathrm{H}, \mathrm{N}-\mathrm{CH}_{2},{ }^{3} \mathrm{~J}=8.0\right.$ $\mathrm{Hz}), 3.90\left(\mathrm{dd}, 1 \mathrm{H}, \mathrm{N}-\mathrm{CH}_{2},{ }^{3} \mathrm{~J}=9.0 \mathrm{~Hz}\right), 5.47\left(\mathrm{t}, 1 \mathrm{H}, \mathrm{O}-\mathrm{CH}_{2},{ }^{3} \mathrm{~J}=8.0 \mathrm{~Hz}\right), 7.35(\mathrm{~m}, 5 \mathrm{H}, \operatorname{arom~} H) .{ }^{13} \mathrm{C}\left\{{ }^{1} \mathrm{H}\right\} \mathrm{NMR}\left(\mathrm{CDCl}_{3}\right.$, $125 \mathrm{MHz}): \delta 14.2\left({ }^{n}\right.$ hexyl $\left.\mathrm{CH}_{3}\right), 22.8\left({ }^{n}\right.$ hexyl $\left.\mathrm{CH}_{2}\right), 26.5\left({ }^{n}\right.$ hexyl $\left.\mathrm{CH}_{2}\right), 27.5\left({ }^{n}\right.$ hexyl $\left.C \mathrm{H}_{2}\right), 31.6\left({ }^{n}\right.$ hexyl $\left.C \mathrm{H}_{2}\right), 44.4\left({ }^{n}\right.$ hexyl $\left.\mathrm{CH}_{2}\right), 52.4\left(\mathrm{~N}_{-} \mathrm{CH}_{2}\right), 74.5(\mathrm{O}-\mathrm{CH}), 125.7(\mathrm{Cm}), 129.0(\mathrm{Co}), 129.1(\mathrm{Cp}), 139.2(\mathrm{Ci}), 158.2(\mathrm{C}=\mathrm{O})$. Anal.: Calcd for $\mathrm{C}_{15} \mathrm{H}_{21} \mathrm{NO}_{2}$ : C, 72.84; H, 8.56; N, 5.66; Found: C, 72.58, H, 8.63, N, 5.82. HREIMS: Calcd. for $\mathrm{C}_{15} \mathrm{H}_{19} \mathrm{NO}_{2}$ : 247.1567; Found: 247.1566.

$\boldsymbol{N}$-iso-propyl-5-phenyloxazolidinone. ${ }^{1} \mathrm{H}$ NMR $\left(\mathrm{CDCl}_{3}, 500 \mathrm{MHz}\right): \delta 1.10\left(\mathrm{~d}, 3 \mathrm{H}\right.$, isopropyl $\left.\mathrm{CH}_{3},{ }^{3} \mathrm{~J}=7.0 \mathrm{~Hz}\right), 1.15(\mathrm{~d}$, $3 \mathrm{H}$, ${ }^{\mathrm{i}}$ propyl $\left.\mathrm{CH}_{3},{ }^{3} \mathrm{~J}=7.0 \mathrm{~Hz}\right), 3.30\left(\mathrm{dd}, 1 \mathrm{H}, \mathrm{N}-\mathrm{CH}_{2},{ }^{3} \mathrm{~J}=8.0 \mathrm{~Hz}\right), 3.82\left(\mathrm{t}, 1 \mathrm{H}, \mathrm{N}-\mathrm{CH}_{2},{ }^{3} \mathrm{~J}=8.5 \mathrm{~Hz}\right), 4.10(\mathrm{~m}, 1 \mathrm{H}, \mathrm{N}-\mathrm{CH})$, $5.41\left(\mathrm{dd}, 1 \mathrm{H}, \mathrm{O}-\mathrm{CH},{ }^{3} \mathrm{~J}=8 \mathrm{~Hz}\right), 7.30(\mathrm{~m}, 5 \mathrm{H}$, arom- $H) .{ }^{13} \mathrm{C}\left\{{ }^{1} \mathrm{H}\right\} \mathrm{NMR}\left(\mathrm{CDCl}_{3}, 125 \mathrm{MHz}\right): \delta 19.7\left(\right.$ propyl $\left.\mathrm{CH}_{3}\right), 20.2$ ('propyl $\left.\mathrm{CH}_{3}\right), 45.1($ propyl $\mathrm{CH}), 47.6\left(\mathrm{~N}_{-} \mathrm{CH}_{2}\right), 74.7(\mathrm{O}-\mathrm{CH}), 125.7(\mathrm{Cm}), 128.9(\mathrm{Co}), 129.1(\mathrm{Cp}), 139.2(\mathrm{Ci}), 157.4$ $(C=\mathrm{O})$. Anal.: Calcd for $\mathrm{C}_{12} \mathrm{H}_{15} \mathrm{NO}_{2}$ : C, 70.22; H, 7.37; N, 6.82; Found: C, 70.20, H, 7.48, N, 6.67. HREIMS: Calcd. for $\mathrm{C}_{12} \mathrm{H}_{15} \mathrm{NO}_{2}$ : 205.1097; Found: 205.1095 .

$N$ - ${ }^{\mathrm{c}}$ hexyl-5-phenyloxazolidinone. An off-white solid (mp 97.3-98.0 $\left.{ }^{\circ} \mathrm{C}\right) .{ }^{1} \mathrm{H}$ NMR $\left(\mathrm{CDCl}_{3}, 500 \mathrm{MHz}\right): \delta 1.06(\mathrm{~m}, 1 \mathrm{H}$, ${ }^{c}$ hexyl $\left.H\right), 1.34\left(\mathrm{~m}, 4 \mathrm{H},{ }^{c}\right.$ hexyl $\left.H\right), 1.62\left(\mathrm{~m}, 1 \mathrm{H},{ }^{c}\right.$ hexyl $\left.H\right), 1.78\left(\mathrm{~m}, 4 \mathrm{H},{ }^{c}\right.$ hexyl $\left.H\right), 3.36\left(\mathrm{dd}, 1 \mathrm{H}, \mathrm{N}-\mathrm{CH}_{2}\right), 3.71(\mathrm{~m}, 1 \mathrm{H}, \mathrm{N}-$ $\mathrm{CH}), 3.86\left(\mathrm{t}, 1 \mathrm{H}, \mathrm{N}-\mathrm{CH}_{2},{ }^{3} \mathrm{~J}=8.5 \mathrm{~Hz}\right), 5.43\left(\mathrm{dd}, 1 \mathrm{H}, \mathrm{O}-\mathrm{CH},{ }^{3} \mathrm{~J}=8.0 \mathrm{~Hz}\right), 7.32(\mathrm{~m}, 5 \mathrm{H}$, arom $H) .{ }^{13} \mathrm{C}\left\{{ }^{1} \mathrm{H}\right\} \mathrm{NMR}\left(\mathrm{CDCl}_{3}\right.$, $125 \mathrm{MHz}$ ): $\delta 25.5$ ('chexyl $\mathrm{CH}_{2}$ ), 25.5 ( ${ }^{c}$ hexyl $\left.\mathrm{CH}_{2}\right), 25.6\left({ }^{c}\right.$ hexyl $\left.\mathrm{CH}_{2}\right), 30.3\left({ }^{c}\right.$ hexyl $\left.\mathrm{CH}_{2}\right), 30.8\left({ }^{c}\right.$ hexyl $\left.C \mathrm{H}_{2}\right), 48.5\left(\mathrm{~N}-\mathrm{CH}_{2}\right)$, $52.8(\mathrm{~N}-\mathrm{CH}), 74.8(\mathrm{O}-\mathrm{CH}), 125.7(\mathrm{Cm}), 128.9(\mathrm{Co}), 129.1(\mathrm{Cp}), 139.3(\mathrm{Ci}), 157.5(\mathrm{C}=\mathrm{O})$. Anal.: Calcd for $\mathrm{C}_{15} \mathrm{H}_{19} \mathrm{NO}_{2}: \mathrm{C}_{\text {, }}$ 73.44; H, 7.81; N, 5.71; Found: C, 73.41, H, 7.82, N, 5.68. HREIMS: Calcd. for $\mathrm{C}_{15} \mathrm{H}_{19} \mathrm{NO}_{2}$ : 245.1410; Found: 245.1409 . 
Figure S2 $\quad$ A) The ${ }^{1} \mathrm{H}$ spectrum of $N^{c}$ 'hexyl-5-phenyloxazolidinone.

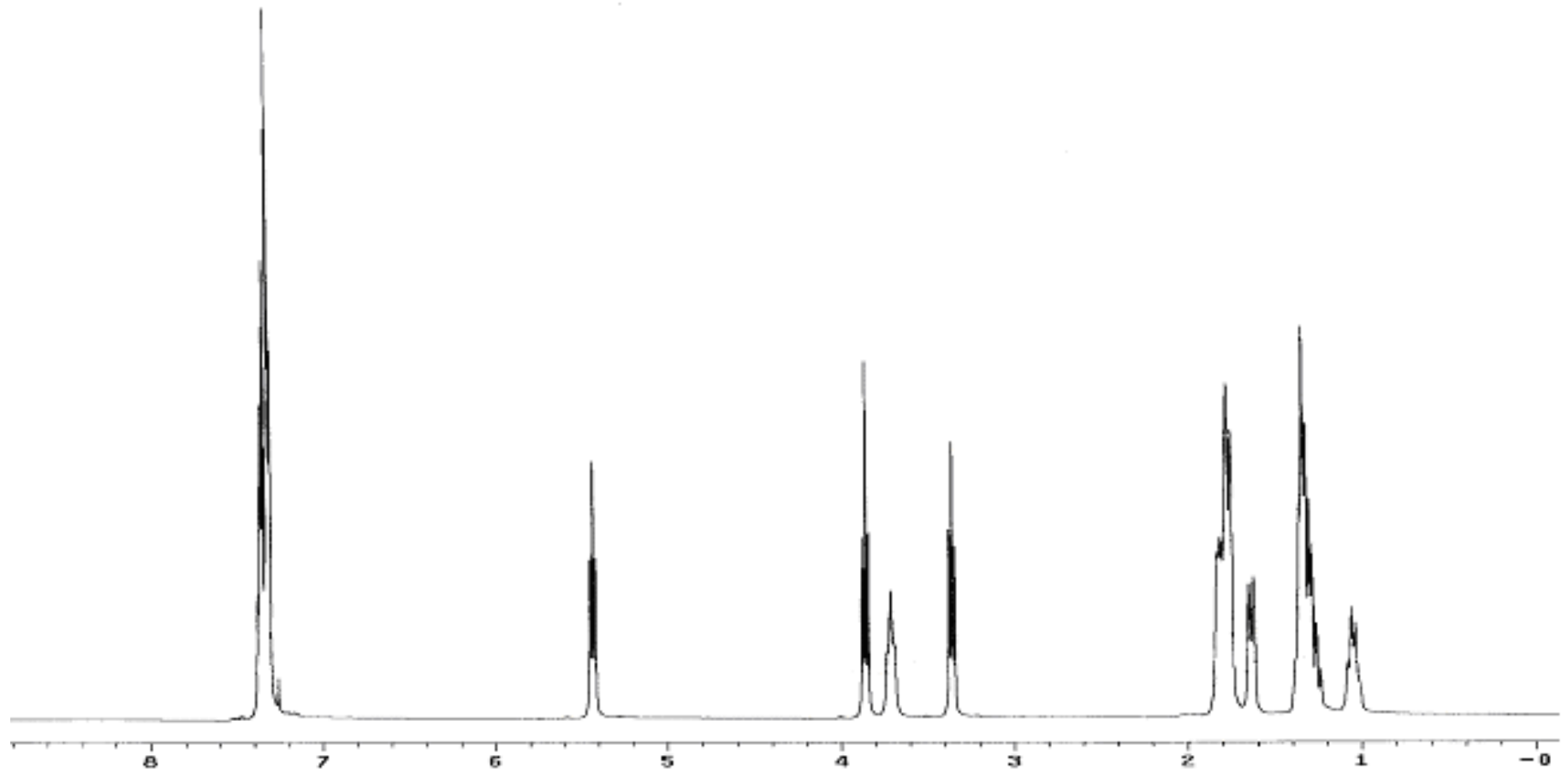

B) The NOESY spectrum of $\mathrm{N}^{c}$ hexyl-5-phenyloxazolidinone.

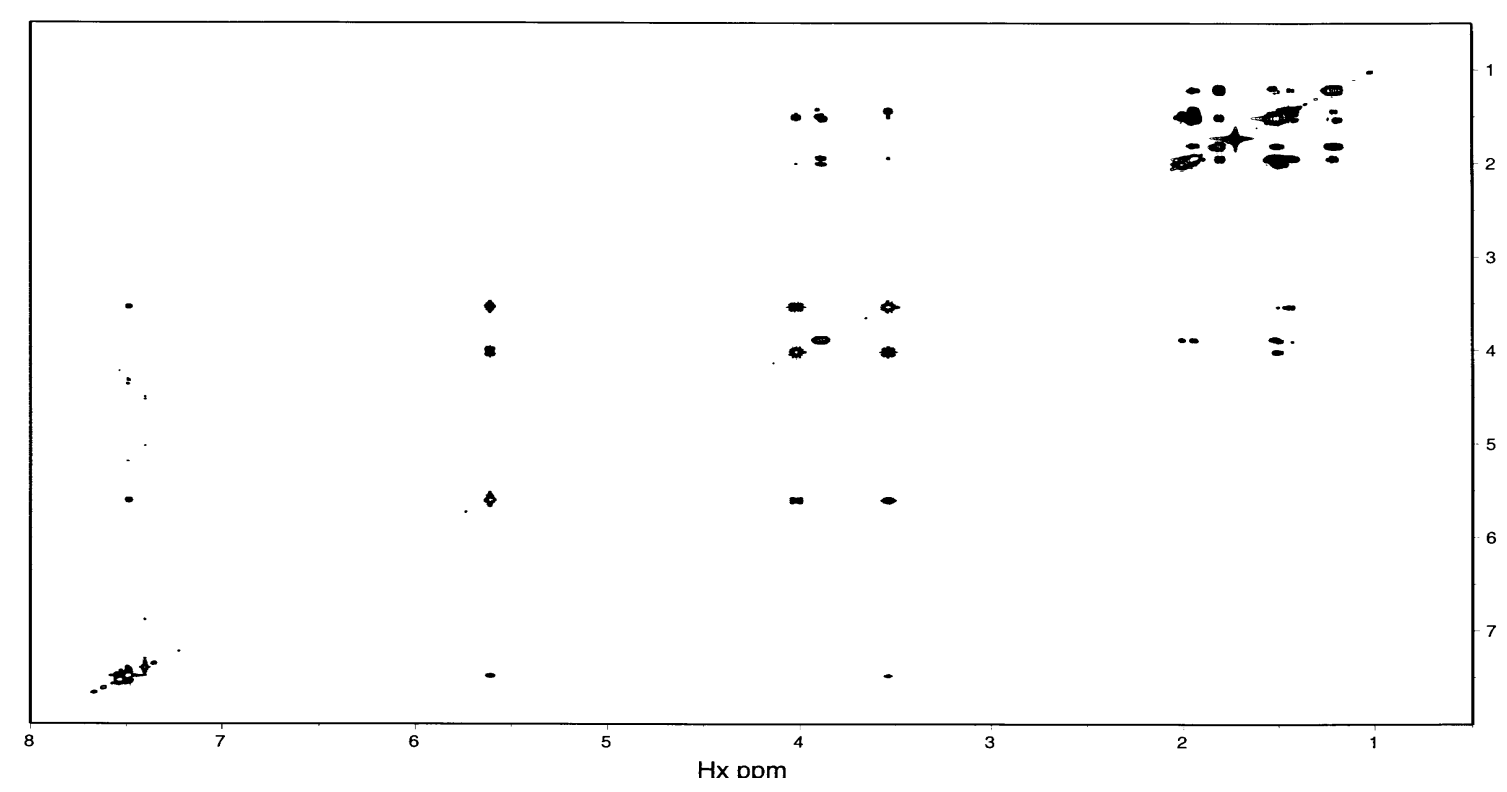


$\boldsymbol{N}$-propyl-hexahydrobenzooxazolidinone: ${ }^{1} \mathrm{H} \mathrm{NMR}\left(\mathrm{CDCl}_{3}, 500 \mathrm{MHz}\right): \delta 0.76\left(\mathrm{t}, 3 \mathrm{H}\right.$, propyl $\left.\mathrm{CH}_{3},{ }^{3} \mathrm{~J}=7.0 \mathrm{~Hz}\right), 1.17$ (m, $1 \mathrm{H},{ }^{c}$ hexyl), $1.33\left(\mathrm{~m}, 3 \mathrm{H},{ }^{c}\right.$ hexyl), $1.37\left(\mathrm{~m}, 2 \mathrm{H}\right.$, propyl $\left.\mathrm{CH}_{2}\right), 1.63\left(\mathrm{~m}, 1 \mathrm{H},{ }^{c}\right.$ hexyl), $1.72\left(\mathrm{~m}, 3 \mathrm{H},{ }^{c}\right.$ hexyl), $2.80(\mathrm{~m}, 1 \mathrm{H}, \mathrm{N}-$ $\left.\mathrm{CH}_{2}\right), 3.21\left(\mathrm{~m}, 1 \mathrm{H}, \mathrm{N}-\mathrm{CH}_{2}\right), 3.53(\mathrm{~m}, 1 \mathrm{H}, \mathrm{N}-\mathrm{CH}), 4.32(\mathrm{~m}, 1 \mathrm{H}, \mathrm{O}-\mathrm{CH}) .{ }^{13} \mathrm{C}\left\{{ }^{1} \mathrm{H}\right\} \mathrm{NMR}\left(\mathrm{CDCl}_{3}, 125 \mathrm{MHz}\right): \delta 11.3$ (propyl $\mathrm{CH}_{3}$ ), 19.7 (propyl $\left.\mathrm{CH}_{2}\right), 19.9$ ( ${ }^{c}$ hexyl), 21.1 ( ${ }^{c}$ hexyl), 25.7 (' hexyl), 27.0 ( ${ }^{c}$ hexyl), $43.4\left(\mathrm{~N}_{-} \mathrm{CH}_{2}\right), 54.2(\mathrm{~N}-\mathrm{CH}), 73.4(\mathrm{O}-$ $C \mathrm{H}), 159.0(C=\mathrm{O})$. Anal.: Calcd for $\mathrm{C}_{10} \mathrm{H}_{17} \mathrm{NO}_{2}$ : C, 65.54; H, 9.35; N, 7.64; Found: C, 65.36, H, 9.46, N, 7.60. HREIMS: Calcd. for $\mathrm{C}_{10} \mathrm{H}_{17} \mathrm{NO}_{2}$ : 183.1254; Found: 183.1249 .

$\boldsymbol{N}$-iso-propyl-5-hexyloxazolidinone. ${ }^{1} \mathrm{H} \mathrm{NMR}\left(\mathrm{CDCl}_{3}, 400 \mathrm{MHz}\right): \delta 0.77\left(\mathrm{t}, 3 \mathrm{H},{ }^{n} \mathrm{hexyl} \mathrm{CH},{ }_{3}^{3} J=6.8 \mathrm{~Hz}\right), \delta 1.06(\mathrm{~d}, 6 \mathrm{H}$, isopropyl $\left.\mathrm{CH}_{3},{ }^{3} \mathrm{~J}=4.8 \mathrm{~Hz}\right), 1.18\left(\mathrm{~m}, 8 \mathrm{H},{ }^{n} \mathrm{hex}{ }^{2-5} \mathrm{CH}_{2}\right) 1.51\left(\mathrm{~m}, 1 \mathrm{H},{ }^{n}\right.$ hexyl $\left.{ }^{1} \mathrm{CH}_{2}\right), 1.61\left(\mathrm{~m}, 1 \mathrm{H},{ }^{n}\right.$ hexyl $\left.{ }^{1} \mathrm{CH}_{2}\right) 2.98(\mathrm{dd}, 1 \mathrm{H}$, $\left.\mathrm{N}-\mathrm{CH}_{2},{ }^{3} \mathrm{~J}=7.2 \mathrm{~Hz}\right), 3.45\left(\mathrm{t}, 1 \mathrm{H}, \mathrm{N}-\mathrm{CH}_{2},{ }^{3} \mathrm{~J}=8.4 \mathrm{~Hz}\right), 3.98(\mathrm{~m}, 1 \mathrm{H}, \mathrm{N}-\mathrm{CH}), 4.37(\mathrm{~m}, 1 \mathrm{H}, \mathrm{O}-\mathrm{CH}) .{ }^{13} \mathrm{C}\left\{{ }^{1} \mathrm{H}\right\} \mathrm{NMR}\left(\mathrm{CDCl}_{3}\right.$, $100 \mathrm{MHz}$ ): $\delta 14.3\left({ }^{n}\right.$ hexyl $\mathrm{CH}_{3}$ ), 19.7 ('propyl $\mathrm{CH}_{3}$ ), 20.1 ('propyl $\left.\mathrm{CH}_{3}\right), 22.7\left({ }^{n}\right.$ hexyl $\left.C \mathrm{H}_{2}\right), 24.7\left({ }^{n}\right.$ hexyl $\left.C \mathrm{H}_{2}\right), 29.1\left({ }^{n}\right.$ hexyl $\mathrm{CH}_{2}$ ), $31.8\left({ }^{n}\right.$ hexyl $\left.\mathrm{CH}_{2}\right), 35.3\left({ }^{n}\right.$ hexyl $\left.\mathrm{CH}_{2}\right), 44.7$ ('propyl $\left.\mathrm{CH}\right), 45.1\left(\mathrm{~N}^{-} \mathrm{CH}_{2}\right), 73.8(\mathrm{O}-\mathrm{CH}), 157.5(\mathrm{C}=\mathrm{O})$. Anal.: Calcd for $\mathrm{C}_{12} \mathrm{H}_{23} \mathrm{NO}_{2}$ : C, 67.57; H, 10.87; N, 6.57; Found: $\mathrm{C}, 67.48, \mathrm{H}, 10.96, \mathrm{~N}, 6.56$. HREIMS: Calcd for $\mathrm{C}_{12} \mathrm{H}_{23} \mathrm{NO}_{2}:$ 213.1723; Found: 213.1726 .

$\boldsymbol{N}$-tert-butyl-5-phenyloxazolidinone ${ }^{1} \mathrm{H}$ NMR $\left(\mathrm{CDCl}_{3}, 500 \mathrm{MHz}\right): \delta 1.41\left(\mathrm{~s}, 9 \mathrm{H},{ }^{t} \mathrm{Butyl} \mathrm{CH}{ }_{3}\right), 3.46\left(\mathrm{t}, 1 \mathrm{H}, \mathrm{N}-\mathrm{CH}_{2},{ }^{3} J=8.5\right.$ $\mathrm{Hz}), 3.96\left(\mathrm{t}, 1 \mathrm{H}, \mathrm{N}-\mathrm{CH}_{2},{ }^{3} \mathrm{~J}=8.5 \mathrm{~Hz}\right), 5.38\left(\mathrm{t}, 1 \mathrm{H}, \mathrm{O}-\mathrm{CH},{ }^{3} \mathrm{~J}=8.0 \mathrm{~Hz}\right), 7.36(\mathrm{~m}, 5 \mathrm{H}, \operatorname{arom}-H) .{ }^{13} \mathrm{C}\left\{{ }^{1} \mathrm{H}\right\} \mathrm{NMR}\left(\mathrm{CDCl}_{3}, 125\right.$ MHz): $\delta 27.6\left({ }^{t} \mathrm{Butyl} \mathrm{CH}_{3}\right), 51.3\left(\mathrm{~N}_{-} \mathrm{CH}_{2}\right), 53.8\left({ }^{t} \mathrm{Butyl} C\right), 73.8(\mathrm{O}-\mathrm{CH}), 125.8(\mathrm{Cm}), 128.9(\mathrm{Co}), 129.1(\mathrm{Cp}), 139.2(\mathrm{Ci})$, 157.0 $(C=\mathrm{O})$. Anal.: Calcd for $\mathrm{C}_{13} \mathrm{H}_{17} \mathrm{NO}_{2}$ : C, 71.21; H, 7.81; N, 6.39; Found: C, 71.25, H, 7.91, N, 6.37. HREIMS: Calcd. for $\mathrm{C}_{13} \mathrm{H}_{17} \mathrm{NO}_{2}$ : 219.1254; Found: 219.1252 .

\section{References.}

(1) Martinez, L. E.; Leighton, J. L.; Carsten, D. H.; Jacobsen, E. N. J. Am. Chem. Soc. 1995, 117, 5897-5898.

(2) Paddock, R. L.; Nguyen, S. T. J. Am. Chem. Soc. 2001, 123, 11498-11499.

(3) Okada, I.; Ichimura, K.; R., S. Bull. Chem. Soc. Jpn. 1970, 43, 1185.

(4) Shibata, I.; Baba, A.; Iwasaki, H.; Matsuda, H. J. Org. Chem. 1986, 51, $2177-2184$. 